\title{
Caracterización de la producción de fibra de vicuña (Vicugna vicugna) en la Región Cusco, Perú
}

\author{
Characterization of the vicuña (Vicugna vicugna) fibre production in the Cusco \\ Region, Perú \\ Joel Pacheco C. ${ }^{1,3}$, Víctor Vélez M. ${ }^{1}$, José Angulo-Tisoc ${ }^{1}$, Sergio Pezo C. ${ }^{1}$, \\ Henry Castelo O. ${ }^{2}$
}

\section{Resumen}

Con el objetivo de describir el diámetro de fibra y el peso de vellón de vicuñas en la Región Cusco, se evaluaron 302 muestras de fibra de vicuñas crías, juveniles y adultas mediante un equipo OFDA $2000 \AA$, colectadas en diferentes capturas programadas (denominadas (Chaccus) bajo dos sistemas de manejo: silvestría y semicautividad. Se registró, además, el peso de 633 vellones diferenciados por edad, sexo y tipo de manejo de los animales. El presente estudio estuvo amparado con la Resolución de Dirección General $\mathrm{N}^{\circ}$ 180-2016-SERFOR/DGGSPFFS. Se determinó la finura promedio de fibra de crías $(13.24 \mu \mathrm{m})$, juveniles $(12.03 \mu \mathrm{m}) \mathrm{y}$ adultos $(12.72 \mu \mathrm{m})$. La finura para machos y hembras fue de 12.99 y $13.53 \mu \mathrm{m}$ en las crías, 12.06 y $12.02 \mu \mathrm{m}$ en los juveniles y 12.88 y $12.58 \mu \mathrm{m}$ en los adultos, respectivamente. El menor diámetro de fibra se presentó en vicuñas juveniles en silvestría $(11.88 \mu \mathrm{m})$ y en la localidad de Canllini $(11.49 \mu \mathrm{m})$. El peso de vellón fue mayor en silvestría $(165.01 \mathrm{~g})$ y en animales adultos $(162.97 \mathrm{~g})$, sin hallar diferencia entre sexos. Se concluye que las mejores características de fibra se encontraron en animales mantenidos en silvestría.

Palabras clave: vicuña; fibra; diámetro; peso de vellón; esquila

\section{AbSTract}

This aim of this study was to describe the fibre diameter and fleece weight of vicuñas in the Cusco Region, Peru. The fibre diameter of 302 fibre samples of young, juveniles and adult vicuñas were evaluated using an OFDA 2000® equipment. The samples were collected in different programmed captures (called Chaccus) under two management

\footnotetext{
${ }^{1}$ Centro de Investigación IVITA, Facultad de Medicina Veterinaria, Universidad Nacional Mayor de San Marcos, Maranganí, Perú

${ }^{2}$ Proyecto de Manejo de Vicuñas, Gobierno Regional del Cusco, Perú

${ }^{3}$ E-mail:jpachecoc@unmsm.edu.pe
}

Recibido: 25 de mayo de 2018

Aceptado para publicación: 24 de octubre de 2018 
systems: wildness and semi-captivity. In addition, 633 fleeces were weighed and differentiated by age, sex and type of management of the animals. The present study was authorized through the Resolution of General Direction N. ${ }^{\circ}$ 180-2016-SERFOR/DGGSPFFS. The average fineness of the fibre of young $(13.24 \mu \mathrm{m})$, juveniles $(12.03 \mu \mathrm{m})$ and adults $(12.72 \mu \mathrm{m})$ was determined. The fineness of fibre for males and females was 12.99 and $13.53 \mu \mathrm{m}$ in young, 12.06 and $12.02 \mu \mathrm{m}$ in juveniles and 12.88 and $12.58 \mu \mathrm{m}$ in adults respectively. The smallest diameter of the fibre was found in juvenile vicunas raised in the wildness $(11.88 \mu \mathrm{m})$ and in the locality of Canllini $(11.49 \mu \mathrm{m})$. The weight of fleece was greater in vicuñas raised in the wildness $(165.01 \mathrm{~g})$ and in adult animals $(162.97 \mathrm{~g})$, and without statistical differences between sexes. It is concluded that the best fibre characteristics were found in animals maintained in the wild.

Key words: vicuna; fibre; diameter; fleece weight; shearing

\section{INTRODUCCIÓN}

La vicuña (Vicugna vicugna mensalis) es una de las cuatro especies de camélidos sudamericanos y es la más pequeña en tamaño; sin embargo, tiene la fibra más fina de sus congéneres, lo cual la hace una especie muy atractiva para su conservación y aprovechamiento racional (Brack, 1980; Quispe et al., 2009). La vicuña habita entre los 3800 a 5200 metros de altitud, principalmente las regiones de puna y cordillera, tiene costumbres polígamas, formando tropillas familiares, siendo ligeramente territoriales (Franklin, 1974; Brack, 1980).

La fibra de la vicuña presenta un diámetro entre 12 a $14 \mu \mathrm{m}$, dependiendo de la edad y condiciones de vida. La fibra se aprovecha en el Perú mediante esquila de animales vivos gracias al Decreto Legislativo N. ${ }^{\circ}$ 653 y desde el año 1994 las poblaciones de vicuñas en el Perú han pasado del Apéndice I al Apéndice II CITES, lo cual permite su producción y comercio internacional (CITES, 2016).

Las características de la fibra han sido ampliamente descritas. En Huancavelica se ha determinado la finura $(13.21 \mu \mathrm{m})$, el peso de vellón (189.7 g) y la longitud de mecha $(30.9 \mathrm{~mm})$ por el sistema óptico OFDA $2000 \AA$
(Quispe et al., 2010). En Puno se obtuvo como promedio $184 \mathrm{~g}$ de peso de vellón (Quispe et al., 2015) y en la puna seca de la Región Tacna se describe $13.0 \mu \mathrm{m}$ de diámetro de fibra y $214.1 \mathrm{~g}$ de peso de vellón (Maquera, 2015). Los diámetros de fibra de vicuñas procedentes de las reservas de Pampa Galeras y Salinas-Aguada Blanca en el Perú para machos jóvenes son de $12.95 \mathrm{y}$ $13.87 \mu \mathrm{m}$, machos adultos de 13.53 y 13.63 $\mu \mathrm{m}$, hembras jóvenes de 13.17 y $13.78 \mu \mathrm{m}, \mathrm{y}$ hembras adultas de 13.83 y $13.83 \mu \mathrm{m}$, respectivamente (Ayala et al., 2003).

Los sistemas de aprovechamiento de vicuñas en los países poseedores de esta especie van desde la silvestría, semicautividad y cautividad; esto último en el Perú es solo para pequeñas cantidades de animales y con fines de exhibición y rescate. La mayor población se encuentra en estado silvestre (silvestría), mientras que una pequeña cantidad es mantenida en semicautividad en extensiones que van desde 500 a más de 1000 ha. Estas unidades son denominados MUS (módulos de uso sustentable) (Quispe et al., 2009). La finura de fibra ha sido, asimismo, evaluada en vicuñas mantenidas en condiciones de semicautividad y silvestría, obteniéndose finuras de 13.27 y $13.19 \mu \mathrm{m}$, respectivamente. Así mismo, se ha determinado que las fibras en las hembras son de menor diámetro que en los machos (Aguilar, 
2010). Por otro lado, Choque et al. (2009) reporta una finura de fibra en la región de Oruro-Bolivia de $14.12 \mu \mathrm{m}$ en crías, 12.87 $\mu \mathrm{m}$ en juveniles y $12.88 \mu \mathrm{m}$ en adultos.

El objetivo del presente estudio fue determinar características de la fibra como diámetro y peso de vellón de acuerdo con la edad, sexo y tipo de manejo en vicuñas de la Región Cusco.

\section{Materiales y Métodos}

El presente estudio está amparado con la autorización de investigación mediante Resolución de Dirección General N. ${ }^{\circ} 180-$ 2016-SERFOR/DGGSPFFS. Las muestras de fibra para determinación de diámetro fueron obtenidas de 302 vicuñas capturadas de diferentes «chaccus» programados en la Región Cusco, llevados a cabo de acuerdo con la Norma Técnica NTP 231.251 (2007), de los cuales 38 fueron crías, 101 juveniles y 163 adultos. La edad fue determinada mediante el tamaño corporal y dentadura (crías: dientes de leche; juveniles: dientes de leche; adultos: 2-6 dientes permanentes). Las vicuñas provinieron de silvestría (109) y semicautividad (193). Se colectaron entre 3 y $4 \mathrm{~g}$ de fibra de la zona del costillar medio de cada individuo, se colocaron en bolsas de polietileno y se llevaron al laboratorio. La determinación del diámetro de la fibra se realizó utilizando el equipo OFDA $2000 \AA$ de La Estación IVITA Maranganí, ubicado en el distrito de Maranganí, Región Cusco, a 3750 msnm.

El peso de vellón fue consignado directamente en el campo, utilizando una balanza de precisión (Metler $\left.{ }^{\circledR}\right)$. Se procesaron 633 registros individuales de pesos de vellón de vicuña, agrupados por tipo de manejo (silvestría, semicautividad), edad (juveniles, adultos) y sexo (machos, hembras).
Los datos fueron analizados utilizando un diseño completamente aleatorizado con un arreglo factorial $2 \times 2 \times 2$ para efectos fijos, organizado en tres factores con sus respectivos niveles (manejo, edad y sexo). Las diferencias entre factores y niveles fueron analizadas para efectos principales y simples a través de la prueba de mínimos cuadrados; además, se procesaron las interacciones manejo x sexo y manejo x edad. Se utilizó el procedimiento GLM y suma de cuadrados tipo III del programa SAS v. 9.0 con p-valor de 0.05 .

\section{Resultados}

Los promedios del diámetro por edad, según las variables manejo, sexo y localidad se encuentran en el Cuadro 1. La determinación del peso de vellón según el tipo de manejo, edad y sexo se encuentra el Cuadro 2 y la interacción del manejo x edad y manejo $\mathrm{x}$ sexo se presenta en el Cuadro 3.

\section{Discusión}

El diámetro promedio de la fibra de vicuña estuvo dentro del rango descrito para la especie (CITES, 2016), similar a lo reportado anteriormente en la región Cusco (Cucho et al., 2012); sin embargo, existen variaciones de acuerdo con los factores evaluados (manejo, edad, sexo), siendo así que el menor valor promedio encontrado en vicuñas juveniles en silvestría $(11.78 \mu \mathrm{m})$ y en el sector de Canllini $(11.49 \mu \mathrm{m})$ fueron inferiores al reporte del CITES (2016).

No existen datos sobre el diámetro de fibra en crías de vicuña en el país, debido a que estos animales no son esquilados; sin embargo, el diámetro promedio obtenido en el presente estudio fue de $13.24 \mu \mathrm{m}$, siendo superior a los diámetros de fibra de juveniles y adultos, posiblemente debido a que las crías 
Cuadro 1. Efecto del tipo de manejo, sexo y localidad sobre el diámetro de fibra $(\mu \mathrm{m})$ en vicuñas de la Región Cusco, Perú (promedios de mínimos cuadrados \pm error estándar de la media)

\begin{tabular}{llccc}
\hline & & Crías & Juveniles & Adultos \\
\hline Manejo & Silvestría & $13.19^{\mathrm{a}} \pm 0.26$ & $11.88^{\mathrm{a}} \pm 0.12$ & $12.65^{\mathrm{a}} \pm 0.11$ \\
& Semicautividad & $13.27^{\mathrm{a}} \pm 0.15$ & $12.24^{\mathrm{b}} \pm 0.10$ & $13.03^{\mathrm{b}} \pm 0.09$ \\
Sexo & Macho & $12.99^{\mathrm{a}} \pm 0.21$ & $12.06^{\mathrm{a}} \pm 0.18$ & $12.88^{\mathrm{a}} \pm 0.20$ \\
& Hembra & $13.53^{\mathrm{b}} \pm 0.22$ & $12.02^{\mathrm{b}} \pm 0.19$ & $12.58^{\mathrm{b}} \pm 0.20$ \\
Localidad & Canllini (S) & $13.07^{\mathrm{a}} \pm 0.24$ & $11.49^{\mathrm{a}} \pm 0.19$ & $12.88^{\mathrm{a}} \pm 0.18$ \\
& T. Estrella (S) & & $12.44^{\mathrm{b}} \pm 0.20$ & $12.67^{\mathrm{a}} \pm 0.14$ \\
& Phacco (S) & & $11.77^{\mathrm{a}} \pm 0.21$ & $11.91^{\mathrm{b}} \pm 0.31$ \\
& Quehue (SC) & & $12.29^{\mathrm{b}} \pm 0.12$ & $13.32^{\mathrm{c}} \pm 0.10$ \\
& Pomacanchi (SC) & $13.07^{\mathrm{a}} \pm 0.22$ & $12.16^{\mathrm{a}} \pm 0.15$ & $12.58^{\mathrm{a}} \pm 0.13$ \\
\hline Promedio & & $13.24 \pm 0.21$ & $12.03 \pm 0.16$ & $12.72 \pm 0.16$ \\
\hline
\end{tabular}

Superíndices dentro de columnas y dentro de cada factor denotan diferencia estadística $(\mathrm{p}<0.05)$ $\mathrm{S}=$ silvestría; $\mathrm{SC}=$ semicautividad

de vicuña presentan un mayor porcentaje de pelos gruesos, formando una evidente doble capa e incrementando el diámetro promedio en general. El diámetro de fibra en las crías fue similar entre aquellas mantenidas en semicautividad y criadas en silvestría, pero hubo diferencia entre sexos, siendo mayor en hembras. En general, el diámetro de fibra en las crías fue inferior al $14.2 \mu \mathrm{m}$ reportado en Bolivia por Choque (2009), siendo esta diferencia posiblemente debida a que el presente estudio se realizó en puna húmeda y cordillera frente al el otro reporte ubicado en puna semidesértica.

El diámetro de fibra de las vicuñas juveniles fue ligeramente inferior al de las vicuñas adultas, de forma similar a lo descrito en Puno (Aguilar, 2010), Ayacucho y Arequipa (Ayala et al., 2003), posiblemente debido a que son animales de primera esquila, similar a lo descrito en alpacas (Tagle, 1990). No obstante, Choque et al. (2009) no encontró diferencia en el diámetro de fibra entre juveniles y adultos, $y$ tampoco hay evi-
Cuadro 2. Peso de vellón influenciado por tipo de sexo, edad y manejo

\begin{tabular}{lll}
\hline Variable & & Peso $(\mathrm{g})$ \\
\hline Sexo & Macho & $147.95^{\mathrm{a}}$ \\
& Hembra & $152.08^{\mathrm{a}}$ \\
Edad & Juvenil & $137.05^{\mathrm{a}}$ \\
& Adulto & $162.97^{\mathrm{b}}$ \\
\multirow{2}{*}{ Manejo } & Silvestría & $165.01^{\mathrm{a}}$ \\
& Semicautividad & $135.00^{\mathrm{b}}$ \\
\hline
\end{tabular}

Superíndices diferentes dentro de cada variable denotan diferencia estadística $(p<0.05)$

dencia de que el número de esquilas afecten el diámetro de fibra de las vicuñas (Cucho et al., 2012).

La finura promedio de fibra de vicuñas juveniles fue menor en las vicuñas mantenidas en silvestría a comparación de las vicuñas en semicautividad $(\mathrm{p}<0.05)$. Así mismo, 
Cuadro 3. Interacción entre manejo y edad y manejo y sexo sobre el peso de vellón

\begin{tabular}{lc}
\hline Interacción & Peso (g) \\
\hline Manejo x edad & \\
$\quad$ Silvestría - Juvenil & $152.18^{\mathrm{a}}$ \\
Silvestría - Adulto & $177.85^{\mathrm{b}}$ \\
Semicautividad - Juvenil & $121.93^{\mathrm{c}}$ \\
$\quad$ Semicautividad - Adulto & $148.09^{\mathrm{a}}$ \\
Manejo x sexo & \\
$\quad$ Silvestría - Macho & $158.85^{\mathrm{a}}$ \\
$\quad$ Silvestría - Hembra & $171.18^{\mathrm{b}}$ \\
$\quad$ Semicautividad - Macho & $137.05^{\mathrm{c}}$ \\
$\quad$ Semicautividad - Hembra & $132.97^{\mathrm{c}}$ \\
\hline Superíndices diferentes dentro de cada \\
interacción denotan diferencia significativa \\
(p<0.05)
\end{tabular}

se observó diferencia entre sexos, aunque Takashima et al. (2017) no encontró diferencia en el diámetro de fibra entre sexos cuando trabajaron con la subespecie $V$. vicugna vicugna. La localidad de muestreo también influencia el diámetro de fibra, tanto en juveniles como en adultos (Cuadro 01; $\mathrm{p}<0.05$ ). Las localidades con mayor finura fueron Canllini y Phaco, ubicadas a $4900 \mathrm{msnm}$ y con agua y bofedales permanentes, lo cual brindaría un mejor tipo de alimentación durante todo el año (Valencia y Gonzales, 2012), no existiendo el efecto estresante del encierro (Siguas et al., 2011).

El diámetro de finura de fibra en los adultos fue inferior a lo descrito en Huancavelica (Quispe, et al., 2010) y Tacna (Maquera, 2015), posiblemente influenciado por la altitud, calidad de la oferta forrajera y la composición florística de estos lugares. Así mismo, las vicuñas adultas mantenidas en silvestría tuvieron un menor diámetro de fibra en comparación con aquellas en semicautividad, lo cual podría ser un indicio de estrés por el manejo de esquilas repetidas (Siguas et al., 2011).
El peso de vellón se encontró por debajo de lo descrito por Quispe et al. (2010) en Huancavelica, Quispe et al. (2015) en Puno, y Maquera (2015) en Tacna, no existiendo diferencias entre sexos. Uno de los factores que podría estar produciendo esta variación de peso sería la mayor altitud promedio de los lugares de captura (4800-5200 m), a diferencia de lo que ocurre en alpacas, donde la altitud parece no influir en el diámetro de la fibra (Braga et al., 2007) y la calidad de la oferta de materia seca desde la pradera, tal como se describe en alpacas (Franco, 2006).

El peso de vellón de animales adultos fue superior al de animales juveniles, lo cual es similar a lo reportado en alpacas (Tagle, 1990), en donde la edad y el número de esquilas incrementaron el peso de vellón. El tipo de manejo influenció el peso del vellón, teniendo vellones más pesados en silvestría, posiblemente por la posibilidad de acceso a pastos de mejor calidad y fuentes de agua, además del estrés causado en las vicuñas en semicautividad por la cercanía de otros grupos familiares y tropillas de machos (Franklin, 1974; Siguas, et al., 2011).

Referente a la interacción sistema de manejo y edad, el mayor peso de vellón se encontró en los adultos en silvestría y de acuerdo a la interacción manejo y sexo, el mayor peso de vellón se encontró en las hembras en silvestría, lo cual indica que el manejo en semicautividad estaría afectando directamente el peso de vellón, posiblemente al afectar el comportamiento social de esta especie y provocando estrés continuo, además de limitar el acceso a pastizales de mejor calidad y fuentes de agua (Miranda et al., 2012; Valencia y Gonzales, 2012).

\section{Agradecimientos}

Los autores agradecen al MVZ Leonidas Coila y al Sr. Flavio Chipana de la Agencia Agraria Canchis, Cusco, por las facilidades brindadas durante la obtención de muestras y datos; así como a los biólogos Jhon Achicahuala y Ronal Rojas del SERFOR Cusco. 


\section{Literatura Citada}

1. Aguilar RM. 2010. Influencia del sistema de manejo en el diámetro y longitud de fibra de vicuñas de la región Puno. Resúmenes III Simposium Internacional de Investigaciones sobre Camélidos Sudamericanos. Arequipa, Perú: DESCO.

2. Ayala J, López M, Chávez J. 2003. Variabilidad del diámetro de fibra de vicuña en dos áreas naturales protegidas del Perú. III Congreso Mundial sobre Camélidos. Potosí, Bolivia.

3. Brack A. 1980. Conservación de la vicuña en el Perú - Información básica. Perú: Ministerio de Agricultura y Alimentación. $33 \mathrm{p}$.

4. Braga W, Leyva V, Cochran R. 2007. The effect of altitude on alpaca (Lama pacos) fiber production. Small Ruminant Res 68: 323-328. doi: 10.1016/ j.smallrumres.2005.11.008

5. Choque S, Cotacallapa F, Ibañez V. 2009. Caracterización física de la fibra de vicuña en los ayllus de la cuenca Lago Poopó de Oruro Bolivia. Allpak'a 14: 37-46.

6. CITES. 2016. Convención sobre el comercio internacional de especies amenazadas de fauna y flora silvestres. Decimoséptima reunión de la conferencia de las partes. Johannesburgo, Sudáfrica. [Internet]. Disponible en: https:// cites.org/sites/default/files/eng/cop/17/ prop/PE_Vicu\%C3\%B1a.pdf

7. Cucho $\bar{H}$, Urquizo D, Ampuero E, Ordoñez, C, Alarcón V, Flores N. 2012. Características de la fibra de vicuña del Centro Experimental La Raya-Cusco. VI Congreso Mundial sobre Camélidos Sudamericanos. Arica, Chile.

8. Franco F. 2006. Efecto del nivel alimenticio sobre el rendimiento y calidad de fibra en alpacas. Tesis de Maestría. Lima, Perú: Univ. Nacional Mayor de San Marcos. 55 p.

9. Franklin W. 1974. The social behaviour of the vicuna. In: Geist V, Walker F (eds). The behavior of ungulates and its relation to management. Alberta, Canada: I.U.C.N. p 477-478.
10. Maquera FE. 2015. Características físicas de la fibra de vicuña en la zona alta andina de Tacna. Rev Investig Altoandin 17: 433-436. doi: 10.18271/ ria.2015.157

11. Miranda E, Cortes A, Miranda E. 2012. Composición de la dieta de la vicuña (Vicugna vicugna) en el altiplano del norte de Chile. VI Congreso Mundial sobre Camélidos Sudamericanos. Arica, Chile.

12. Norma Técnica Peruana NTP 231.351. 2007. Fibra de vicuña. Proceso de esquila mecánica de la vicuña. NTP 231.351 - R.0094-2007/INDECOPI-CTR.

13. Quispe EC, Rodríguez TC, Iñiguez LR, Mueller JP. 2009. Producción de fibra de alpaca, llama, vicuña y guanaco en Sudamérica. Animal Genetic Resources Information 45: 1-14 doi: 10.1017/S1014233909990277

14. Quispe EC, Ramos H, Mayhua P, Alonso L. 2010. Fibre characteristics of vicuña (Vicugna vicugna mensalis). Small Ruminant Res 93: 64-66 doi: 10.1016/j.smallrumres.2010.03.019

15. Quispe JE, Butron B, Quispe DM, Arratia M. 2015. Producción de fibra de vicuña en semicautiverio y silvestria: tendencia, características y situación actual en la Región Puno. Rev Investig Altoandin 17: 369-378. doi: 10.18271/ ria.2015.148

16. Siguas O, Olazábal J, Paucar R, Bonacic C. 2011. Respuesta de estrés de captura en vicuñas silvestres (Vicugna vicugna Thomas, 1917) en condiciones de puna peruana. VII Congreso de la Asociación Latinoamericana de Especialistas en Pequeños Rumiantes y Camélidos Sudamericanos ALEPRyCS. Huancavelica, Perú.

17. Tagle S. 1990. Crecimiento y finura de fibra en alpacas Huacaya y Suri durante el año de 1989. Tesis de Médico Veterinario y Zootecnista. Puno, Perú: Univ. Nacional del Altiplano. $69 \mathrm{p}$. 
18. Takashima C, Dionicio A, Carfagnini M, Saralegui S, Di-Mauro S, Pacheco C, Marino P. 2017. Medición de finura y longitud de fibra de vicuña obtenida en esquilas comunitarias en la Provincia de Jujuy, Argentina. Rev Invest Altoandin 19: 187-194.
19. Valencia DI, Gonzáles BA. 2012. Uso de habitat de vicuñas y llamas en el Salar de Tara, Región de Antofagasta. VI Congreso Mundial sobre Camélidos Sudamericanos. Arica, Chile. 